\title{
A Rare Case of Local Recurrence Following Curative Endoscopic Submucosal Dissection of Intramucosal Differentiated-Type Gastric Cancer
}

\author{
Hideaki Kawabata ${ }^{\text {a, c }}$, Yukino Kawakatsu ${ }^{a}$, Katsutoshi Yamaguchia, Yuki Ueda ${ }^{\text {a }}$, \\ Yuji Okazaki a , Misuzu Hitomia, Masatoshi Miyata ${ }^{a}$, Shigehiro Motoia, \\ Yasuyuki Enoki ${ }^{\text {b }}$, Satohiro Minamikawa ${ }^{\mathrm{b}}$
}

\begin{abstract}
A 78-year-old man underwent endoscopic submucosal dissection (ESD) of early gastric cancer (EGC) (type 0-IIa) in the anterior wall of the antrum. En bloc resection was achieved. The histopathological examination revealed very well-differentiated tubular adenocarcinoma (tub1) of $30 \times 22 \mathrm{~mm}$ in size, confined to the mucosa. No lymphovascular invasion or ulceration was observed, and there was no undifferentiated-type component and the margins were tumor-free. Therefore, this lesion fulfilled the eCuraA criteria. Two years after ESD, esophagogastroduodenoscopy revealed an irregular, slightlydepressed lesion within the post-ESD scar. Tubular adenocarcinoma was suspected based on histopathological examination of a biopsy specimen. The tumor was resected by ESD. A histopathological examination revealed well-differentiated tubular adenocarcinoma (tub1) of $6 \times 4 \mathrm{~mm}$ in size, confined to the mucosa. No lymphovascular invasion was detected and the margins were tumor-free. These findings indicated a curative resection. Recurrence following a curative ESD of an intramucosal differentiated-type EGC which fulfilled the eCuraA criteria is rare. Careful endoscopic observation using magnifying narrow band imaging (NBI) is needed after ESD, even when curative resection is achieved.
\end{abstract}

Keywords: Early gastric cancer; Endoscopic submucosal dissection; eCuraA

\section{Introduction}

Endoscopic submucosal dissection (ESD) has been widely indicated and performed as a minimally invasive treatment for

Manuscript submitted February 12, 2019, accepted March 7, 2019

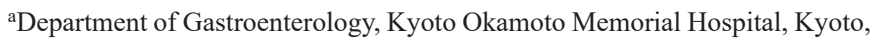
Japan

bDepartment of Pathology, Kyoto Okamoto Memorial Hospital, Kyoto, Japan ${ }^{\mathrm{c} C}$ Corresponding Author: Hideaki Kawabata, Department of Gastroenterology, Kyoto Okamoto Memorial Hospital, 58 Nishinokuchi, Sayama, Kumiyamacho, Kuze-gun, Kyoto 613-0034, Japan. Email: hkawabata@okamoto-hp.or.jp

doi: https://doi.org/10.14740/gr1159 early gastric cancer (EGC). In the Japanese Gastric Cancer Treatment Guideline (fifth version) revised in 2018 [1], the absolute indications for curative endoscopic resection of EGC were expanded as follows: tumors clinically diagnosed as intramucosal (T1a) differentiated-type cancer (well or moderately differentiated adenocarcinoma or papillary adenocarcinoma) without ulceration (UL(-)) regardless of size and intramucosal (T1a) differentiated-type cancer measuring $\leq 3 \mathrm{~cm}$ in diameter with ulceration $(\mathrm{UL}(+))$. Fulfilling the definition of curative resection A (eCuraA; the highest curability achievable by endoscopic resection) required the fulfillment of all the following criteria on a histological examination of the resected specimen: 1) En bloc resection; 2) Differentiated-type dominant adenocarcinoma; 3) pT1a; 4) UL(-) tumor of any size or UL(+) tumor of $\leq 3 \mathrm{~cm}$ in size; 5) Tumor-free at both horizontal and vertical margins; and 6) No lymphatic or vascular involvement. Favorable outcomes including a low incidence of long-term recurrence $(0-0.2 \%)$ have been reported after curative ESD in large studies [2-7]. All recurrent cases reported in these studies involved lesions with slight submucosal invasion (SM1) or were classified as non-curative resection.

We herein report a rare case of local recurrence following curative ESD of intramucosal differentiated-type EGC.

\section{Case Report}

A 78-year-old man underwent ESD of EGC (type 0-IIa) in the anterior wall of the antrum in October 2016. En bloc resection was achieved (Fig. 1a, b). The histopathological examination of the resected specimen revealed very well-differentiated tubular adenocarcinoma (tub1) of $30 \times 22 \mathrm{~mm}$ in size, confined to the mucosa. No lymphovascular invasion or ulceration was observed, there was no undifferentiated-type (namely, poorly differentiated adenocarcinoma, signet ring cell carcinoma or mucinous adenocarcinoma) component, and the margins were tumor-free (Fig. 2a-c). This lesion fulfilled the absolute pathological criteria for eCuraA. The mucosa adjacent to the cancer on the ESD specimen showed atrophy and intestinal metaplasia. The patient's serum was negative for Helicobacter pylori antibodies, despite the gastric mucosa being broadly atrophic endoscopically. 


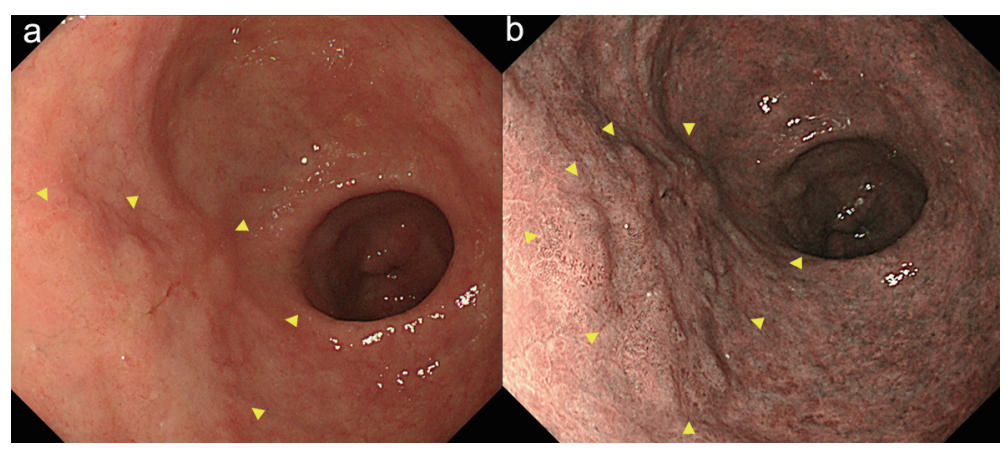

Figure 1. Endoscopy showed a type 0-lla early gastric cancer in the anterior wall of the antrum (arrow heads). (a) white light image, and (b) NBI image. NBI: narrow band imaging.

a
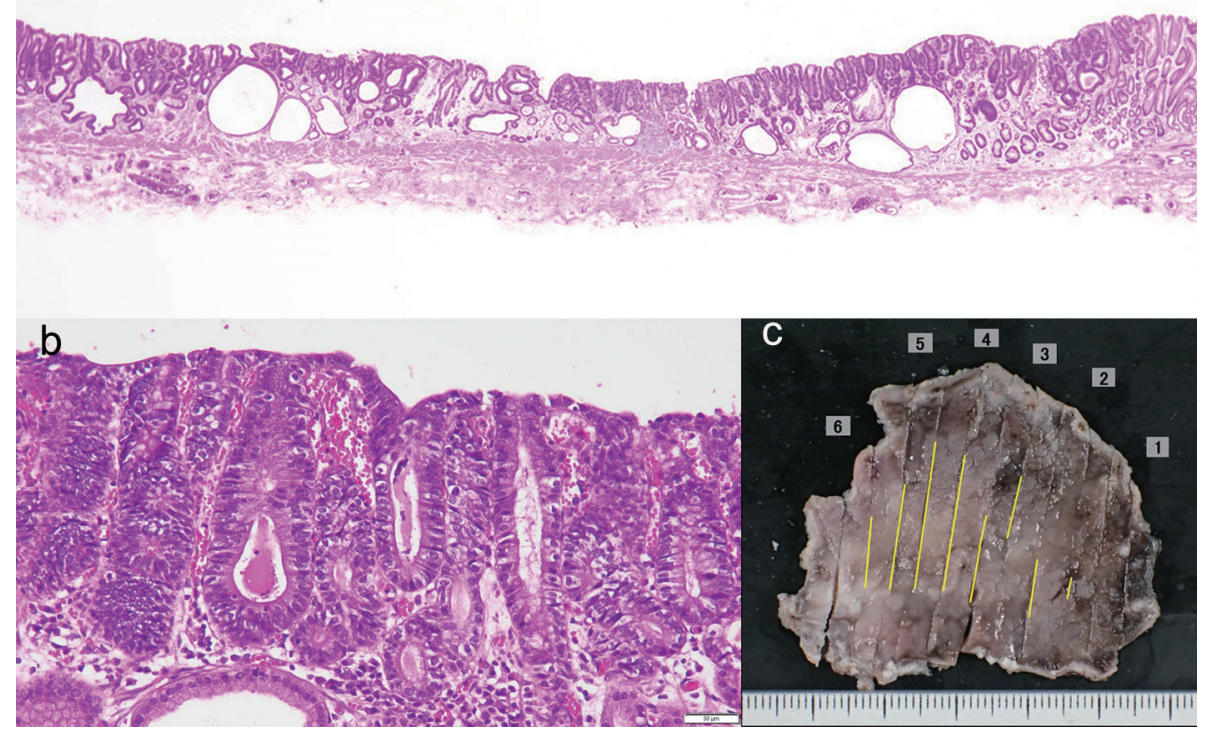

Figure 2. (a-c) The histopathological examination of the resected specimen revealed very well-differentiated tubular adenocarcinoma (tub1) of $30 \times 22 \mathrm{~mm}$ in size, confined to the mucosa. No lymphovascular invasion or ulceration was observed, there was no undifferentiated-type component, and the margins were tumor-free.

Follow-up esophagogastroduodenoscopy performed in November 2018 (when the patient was free of symptoms) revealed an irregular, slightly-depressed lesion within the post-
ESD scar (Fig. 3a, b). The lesion showed irregular microvessels on magnifying narrow band imaging (NBI) (Fig. 3c). Tubular adenocarcinoma was suspected based on the histopathological

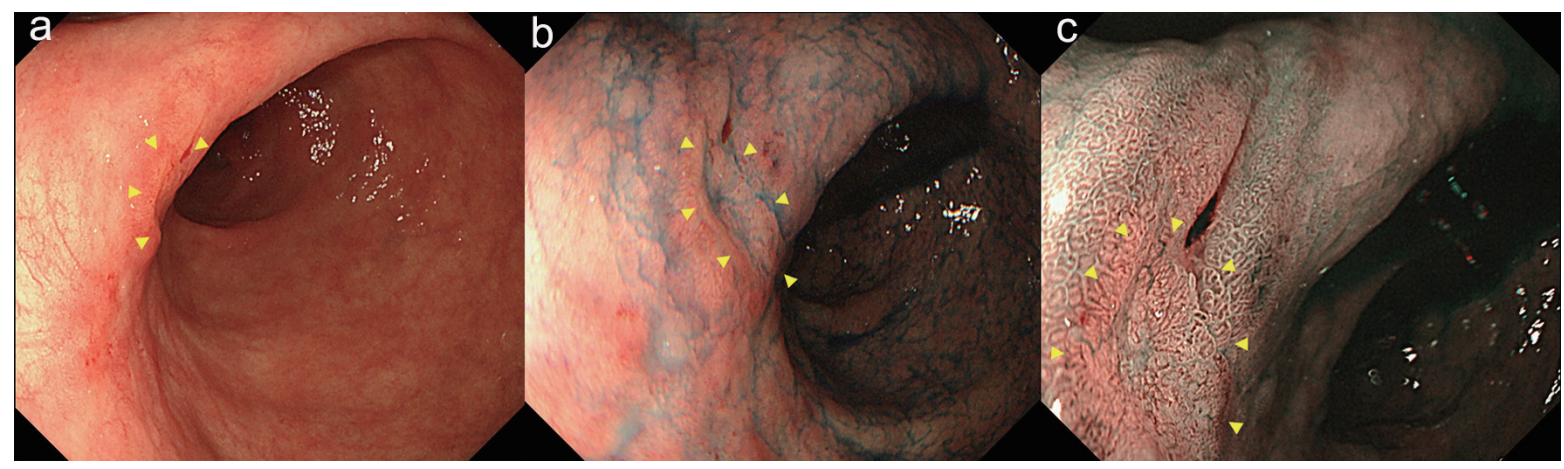

Figure 3. The follow-up endoscopy showed an irregular, slightly-depressed lesion within the post-ESD scar (arrow heads) (a, b). The lesion showed irregular microvessels on magnifying NBI (arrow heads) (c). 
examination of a biopsy specimen. Endoscopic ultrasonography revealed broad hypoechoic fibrotic lesion up to the shallow submucosal layer (Fig. 4). CT revealed no signs of metastasis. As the demarcation line of the tumor was unclear, we resected the tumor including whole post-ESD scar by ESD. A histopathological examination of the resected specimen revealed well-differentiated tubular adenocarcinoma (tub1) of 6 $\times 4 \mathrm{~mm}$ in size, confined to the mucosa. No lymphovascular invasion was detected and the margins were tumor-free (Fig. 5a-c). These findings indicated a curative resection. Extensive thick fibrosis was observed in the submucosa. No procedurerelated complications were observed, and the postoperative course was uneventful.

\section{Discussion}

We experienced a case of local recurrence following curative ESD of an intramucosal differentiated-type EGC that fulfilled the eCuraA criteria. We found two other cases of recurrence after ESD that fulfilled the eCuraA criteria. Fujii et al [8] reported a case of lymph node metastasis after curative ESD of a moderately differentiated intramucosal EGC with a maximum diameter of $22 \mathrm{~mm}$ accompanied by an ulcer that widely contacted the muscularis mucosae. Hanaoka et al [9] reported a case of nodal recurrence and liver metastasis after curative ESD of a moderately differentiated-dominant intramucosal EGC, intermingled with poorly differentiated adenocarcinoma in some regions. Tsuji et al [10] reported that the risk factors for lymph node metastasis from intramucosal EGC were as follows: 1) Histologically mixed type; 2) Disappearance of the

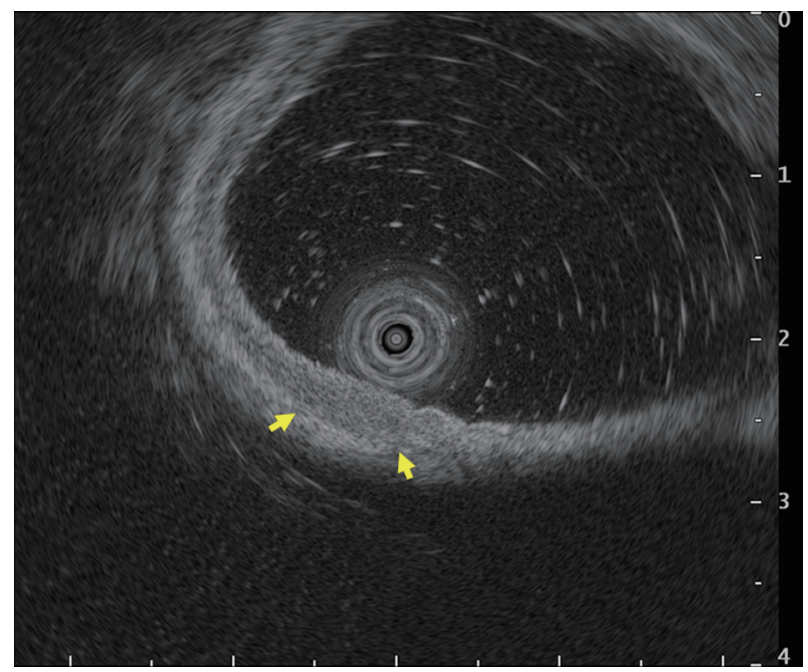

Figure 4. Endoscopic ultrasonography revealed broad hypoechoic fibrotic lesion up to the shallow submucosal layer (arrows).

muscularis mucosae at the top of invasion; 3 ) Length of attachment of cancer cells to the muscularis mucosae $\geq 10 \mathrm{~mm}$; 4) p53 protein positively; and 5) Vascularity of cancer surrounding tissue $\geq 30 / \mathrm{mm}^{2}$. The lesions in two of the previously reported cases contained one of these risk factors and attention should have been paid to nodal recurrence. On the other hand, our case had no nodal recurrence and the lesion fulfilled none of the risk factors for nodal recurrence, although p53 was not investigated. One possible cause of local recurrence in our case is the existence of another latent or minute cancer adjacent to
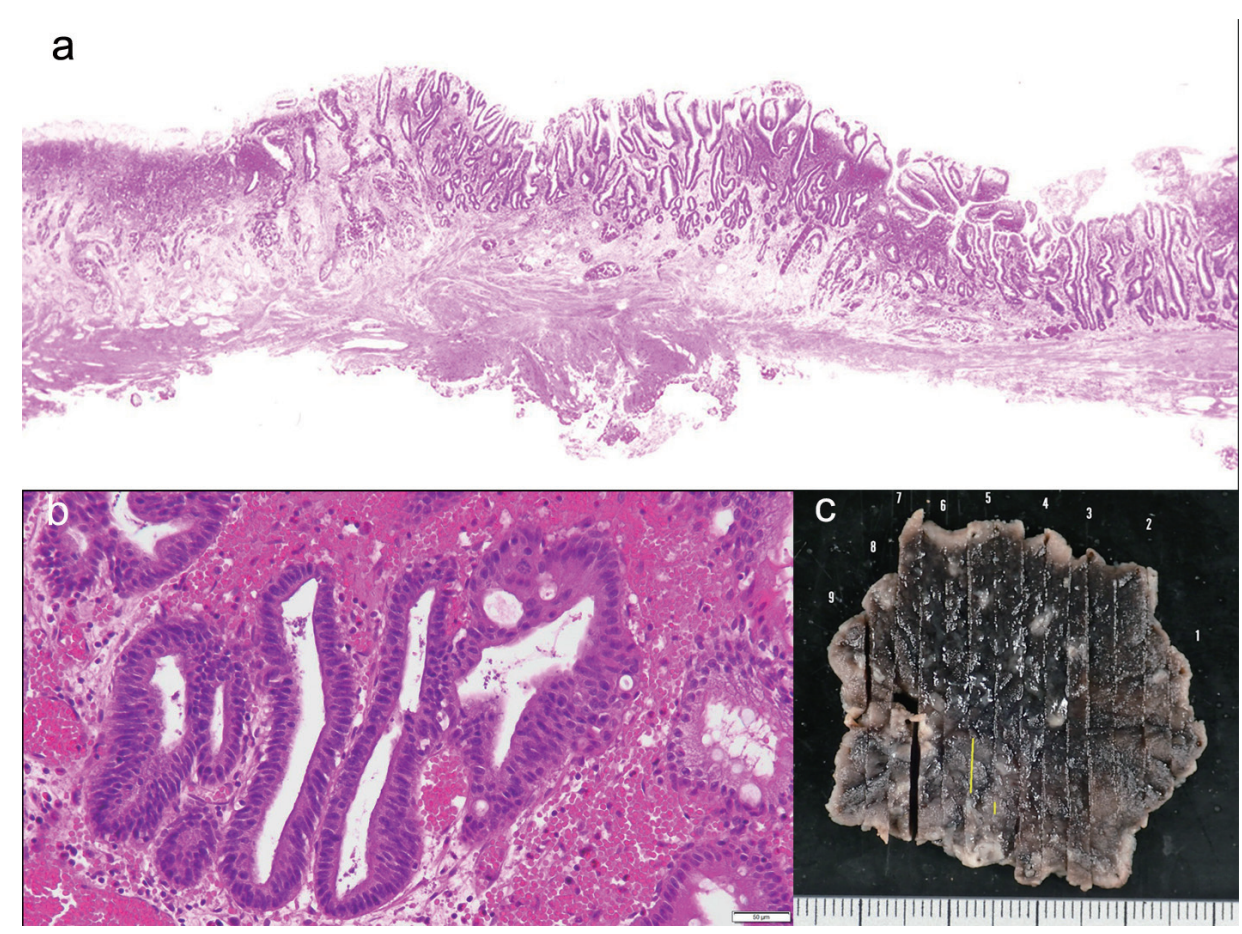

Figure 5. (a-c) A histopathological examination of the resected specimen revealed well-differentiated tubular adenocarcinoma (tub1) of $6 \times 4 \mathrm{~mm}$ in size, confined to the mucosa. No lymphovascular invasion was detected and the margins were tumor-free. 
the primary cancer, which was involved in the scar after ESD. Although no other EGC was detected adjacent to the primary tumor in our review of past endoscopic images, broad mucosal atrophy, which is a risk factor of GC, was observed around the tumor and it is not rare to encounter synchronous multiple gastric cancers (the reported incidence is $4.4-5.7 \%[11,12]$ ).

In our case, magnifying endoscopy with NBI was useful for diagnosing the recurrent cancer. This modality enabled us to find the minute irregularity of the microsurface and microvascular structure in the scar after ESD. Magnifying NBI should be performed in routine esophagogastroduodenoscopy because it improves diagnostic accuracy in cases of EGC $[13,14]$.

In conclusion, we experienced a rare case of local recurrence following curative ESD of an intramucosal differentiated-type EGC that fulfilled the eCuraA criteria. Careful endoscopic observation using magnifying NBI is needed after ESD, even when curative resection is achieved.

\section{Conflict of Interest}

The authors declare no conflict of interest.

\section{References}

1. Japanese Gastric Cancer Association. Treatment guidelines for gastric cancer in Japan. 5th ed. Tokyo: Kanehara; 2018 (in Japanese).

2. Goto O, Fujishiro M, Kodashima S, Ono S, Omata M. Outcomes of endoscopic submucosal dissection for early gastric cancer with special reference to validation for curability criteria. Endoscopy. 2009;41(2):118-122.

3. Isomoto H, Shikuwa S, Yamaguchi N, Fukuda E, Ikeda K, Nishiyama H, Ohnita K, et al. Endoscopic submucosal dissection for early gastric cancer: a large-scale feasibility study. Gut. 2009;58(3):331-336.

4. Kosaka T, Endo M, Toya Y, Abiko Y, Kudara N, Inomata $\mathrm{M}$, Chiba $\mathrm{T}$, et al. Long-term outcomes of endoscopic submucosal dissection for early gastric cancer: a singlecenter retrospective study. Dig Endosc. 2014;26(2):183191.

5. Tanabe S, Ishido K, Higuchi K, Sasaki T, Katada C, Azuma M, Naruke A, et al. Long-term outcomes of endoscopic submucosal dissection for early gastric cancer: a retrospec- tive comparison with conventional endoscopic resection in a single center. Gastric Cancer. 2014;17(1):130-136.

6. Suzuki H, Oda I, Abe S, Sekiguchi M, Mori G, Nonaka $\mathrm{S}$, Yoshinaga S, et al. High rate of 5-year survival among patients with early gastric cancer undergoing curative endoscopic submucosal dissection. Gastric Cancer. 2016;19(1):198-205.

7. Hasuike N, Ono H, Boku N, Mizusawa J, Takizawa K, Fukuda H, Oda I, et al. A non-randomized confirmatory trial of an expanded indication for endoscopic submucosal dissection for intestinal-type gastric cancer (cT1a): the Japan Clinical Oncology Group study (JCOG0607). Gastric Cancer. 2018;21(1):114-123.

8. Fujii H, Ishii E, Tochitani S, Nakaji S, Hirata N, Kusanagi H, Narita M. Lymph node metastasis after endoscopic submucosal dissection of a differentiated gastric cancer confined to the mucosa with an ulcer smaller than $30 \mathrm{~mm}$. Dig Endosc. 2015;27(1):159-161.

9. Hanaoka N, Tanabe S, Higuchi K, Sasaki T, Nakatani K, Ishido K, Ae T, et al. A rare case of histologically mixedtype intramucosal gastric cancer accompanied by nodal recurrence and liver metastasis after endoscopic submucosal dissection. Gastrointest Endosc. 2009;69(3 Pt 1):588-590.

10. Tsujio G, Kashiwagi S, Asano Y, Goto W, Takada K, Morisaki T, Noda S, et al. [A case of T1a breast cancer with axillary lymph node metastasis]. Gan To Kagaku Ryoho. 2017;44(12):1059-1061.

11. Jeong SH, An J, Kwon KA, Lee WK, Kim KO, Chung JW, Kim YJ, et al. Predictive risk factors associated with synchronous multiple early gastric cancer. Medicine (Baltimore). 2017;96(26):e7088.

12. Kodera Y, Yamamura Y, Torii A, Uesaka K, Hirai T, Yasui $\mathrm{K}$, Morimoto $\mathrm{T}$, et al. Incidence, diagnosis and significance of multiple gastric cancer. Br J Surg. 1995;82(11):15401543.

13. Yoshimizu S, Yamamoto Y, Horiuchi Y, Omae M, Yoshio $\mathrm{T}$, Ishiyama A, Hirasawa T, et al. Diagnostic performance of routine esophagogastroduodenoscopy using magnifying endoscope with narrow-band imaging for gastric cancer. Dig Endosc. 2018;30(1):71-78.

14. Yu H, Yang AM, Lu XH, Zhou WX, Yao F, Fei GJ, Guo $\mathrm{T}$, et al. Magnifying narrow-band imaging endoscopy is superior in diagnosis of early gastric cancer. World J Gastroenterol. 2015;21(30):9156-9162. 